\title{
IdeAs
}

Idées d'Amériques

$4 \mid 2013$

Crises et effets de crise dans les Amériques

\section{América latina frente a la crisis económica internacional : buena resistencia global y diversidad de situaciones nacionales}

L'Amérique latine face à la crise économique internationale : bonne résistance globale et diversité de situations nationales

Latin America facing the international economic crisis : good overall resilience and diversity of national situations

\section{Carlos Quenan}

\section{OpenEdition}

Journals

Édition électronique

URL : https://journals.openedition.org/ideas/780

DOI : $10.4000 /$ ideas. 780

ISSN : $1950-5701$

Éditeur

Institut des Amériques

Référence électronique

Carlos Quenan, « América latina frente a la crisis económica internacional : buena resistencia global y diversidad de situaciones nacionales », IdeAs [En ligne], 4 | 2013, mis en ligne le 15 octobre 2013, consulté le 18 octobre 2022. URL : http://journals.openedition.org/ideas/780 ; DOI : https://doi.org/ 10.4000/ideas.780

Ce document a été généré automatiquement le 18 octobre 2022

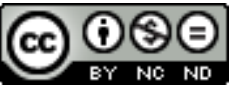

Creative Commons - Attribution - Pas d'Utilisation Commerciale - Pas de Modification 4.0 International - CC BY-NC-ND 4.0

https://creativecommons.org/licenses/by-nc-nd/4.0/ 


\title{
América latina frente a la crisis económica internacional : buena resistencia global y diversidad de situaciones nacionales
}

\author{
L'Amérique latine face à la crise économique internationale : bonne résistance \\ globale et diversité de situations nationales \\ Latin America facing the international economic crisis : good overall resilience \\ and diversity of national situations
}

Carlos Quenan

\section{NOTE DE L'ÉDITEUR}

Cet article est traduit du français par Marta Gómez

\section{Introducción}

1 Tras dos décadas caracterizadas por el recrudecimiento de las crisis monetarias y financieras y un crecimiento mediocre y volátil, América Latina ha experimentado en los años 2000 un fuerte dinamismo de la actividad económica y ha reducido su vulnerabilidad financiera. Esto ha permitido que la región resista bien frente a la crisis económica internacional desatada en 2007, que la afectó más por el canal comercial que a través del canal financiero (Ocampo, 2009). Así, tras la recesión - moderada a fin de cuentas - de 2009, se ha producido una recuperación bastante sólida y, en 2010, la región latinoamericana ha registrado un crecimiento superior al $6 \%$.

2 Nuestro objetivo aquí es abordar los efectos que ha tenido en América Latina el agravamiento de la crisis económica internacional en 2008-2009. En términos generales, 
la región se ha mostrado mejor preparada que en el pasado para afrontar situaciones adversas y ha resistido relativamente bien frente a la crisis internacional. Pero más allá de las características comunes al conjunto de la región, la crisis ha puesto de manifiesto una gran diversidad de situaciones nacionales. Así, pueden distinguirse los países que han mostrado una gran capacidad de resistencia frente a las perturbaciones y otros, por el contrario, cuyas debilidades y fragilidades han sido reveladas por la crisis.

\section{Años 2000 : crecimiento económico, mejora de la gestión macroeconómica y disminución de la vulnerabilidad financiera}

Durante la primera década del siglo XXI, la región latinoamericana experimentó un crecimiento, en muchos aspectos, inédito. En los años 2000, la expansión del crecimiento estuvo acompañada de superávits por cuenta corriente, al menos hasta 2007. Este fenómeno ha sido excepcional si se tienen en cuenta los resultados registrados por las economías latinoamericanas en las últimas décadas (Gráfico 1). En efecto, durante las fases de expansión de la actividad económica, una mayor progresión de las importaciones con respecto a las exportaciones se traducía habitualmente en un déficit exterior que podía bloquear el crecimiento si las condiciones de financiación empeoraban. Por otra parte, desde los años 70, las fases en las que las economías de la región experimentaron una aceleración del crecimiento - segunda mitad de los años 70 y parte de los 90 - coincidieron con importantes entradas de capitales (Gráfico 2), que constituyeron el preludio de graves crisis monetarias y financieras.

4 Por el contrario, en la fase 2003-2008, el aumento de las exportaciones de materias primas y el alza de los precios de estos productos, sostenidos por el dinamismo de la demanda asiática - y sobre todo, china - permitieron que los países de la región - y en particular los países de América del Sur, cuya especialización internacional conlleva grandes ventajas comparativas en el sector primario $^{1}$ - evitaran la aparición de presiones financieras externas. Más que el ciclo financiero (entradas o salidas netas de capital), lo que estuvo fuertemente asociado al comportamiento de la actividad económica en el periodo 2003-2008 fue la evolución favorable de los términos de intercambio. 
Gráfico 1 : América Latina : PIB real (\% interanual, eje horizontal) y saldo corriente ( $\%$ del PIB, eje vertical)

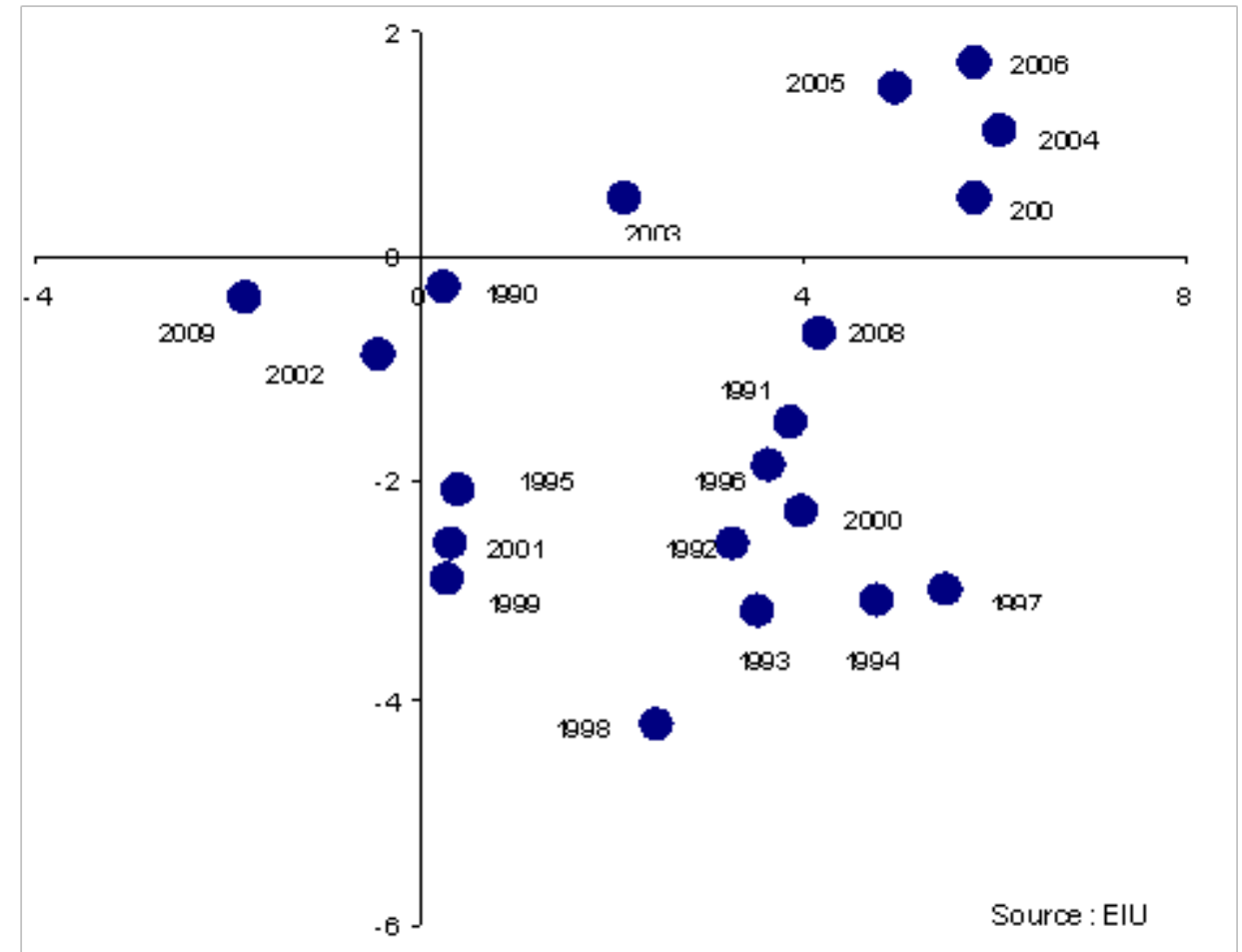

Gráfico 2 : América Latina : PIB real y flujos de capitales

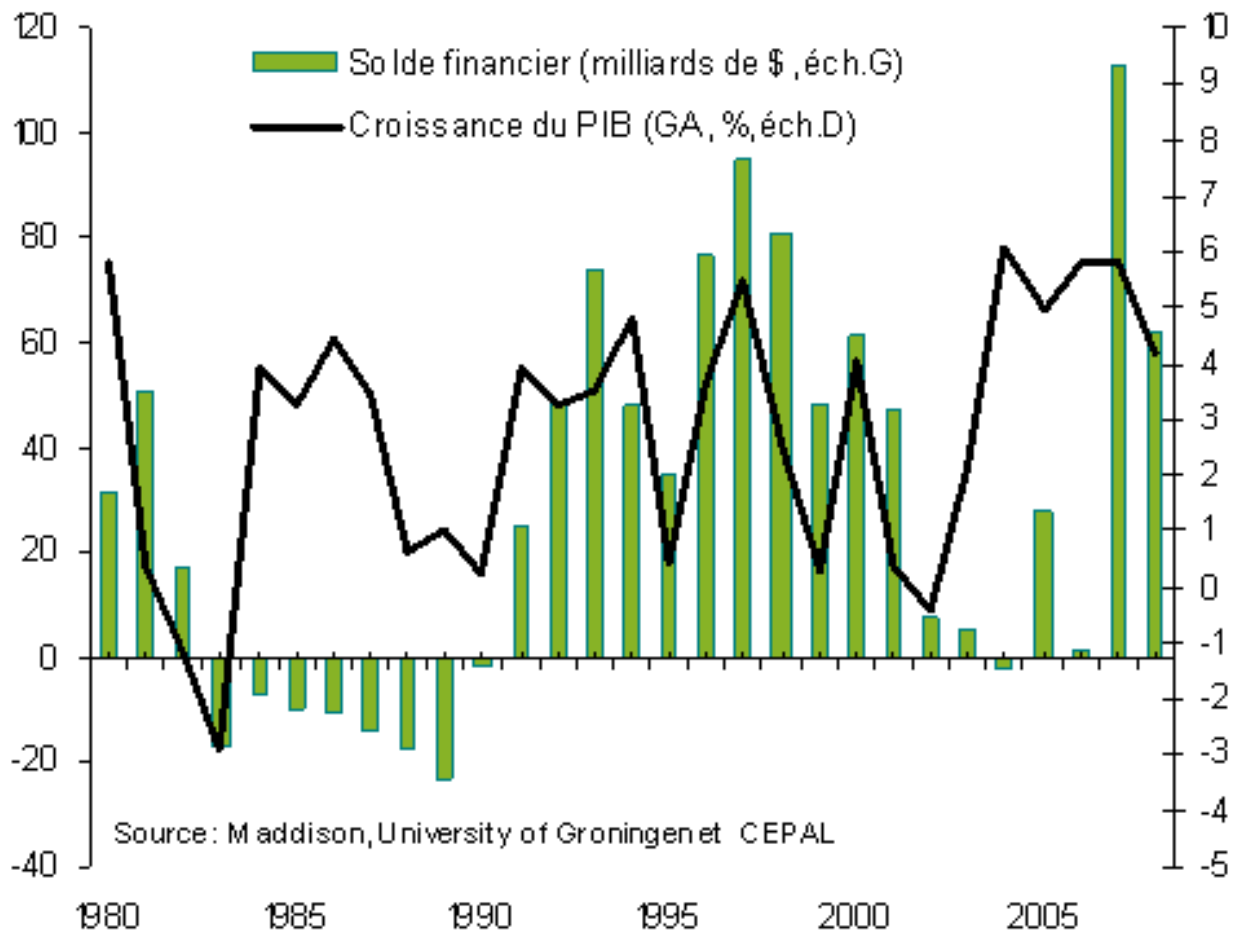

5 Si bien en los años 2000 es incuestionable la incidencia de un entorno internacional favorable en la recuperación de las economías latinoamericanas, no hay que obviar el 
peso de la dinámica interna. Si abordamos esta cuestión desde la perspectiva de la contribución al crecimiento de los distintos componentes del PIB (consumo privado de los hogares, gastos de consumo de las administraciones públicas, inversión, exportaciones), vemos cómo el peso de la demanda exterior (bruta) explica al menos el $40 \%$ del crecimiento económico de países como México, Chile, Bolivia o Brasil en el ciclo de crecimiento 2003-2008, considerando únicamente los efectos "directos $»^{2}$. En otros países como Argentina o Venezuela, que entre 2003 y 2008 experimentaron un incremento bastante notable del PIB real (49,8\% y $62,8 \%$ respectivamente), el crecimiento estuvo ligado en gran medida a la recomposición de la demanda interna tras las severas crisis que vivieron estos países a principios de la década de los 2000 (Tabla 1 y Gráfico 3).

Tabla 1 : Oferta y demanda agregadas a precios constantes

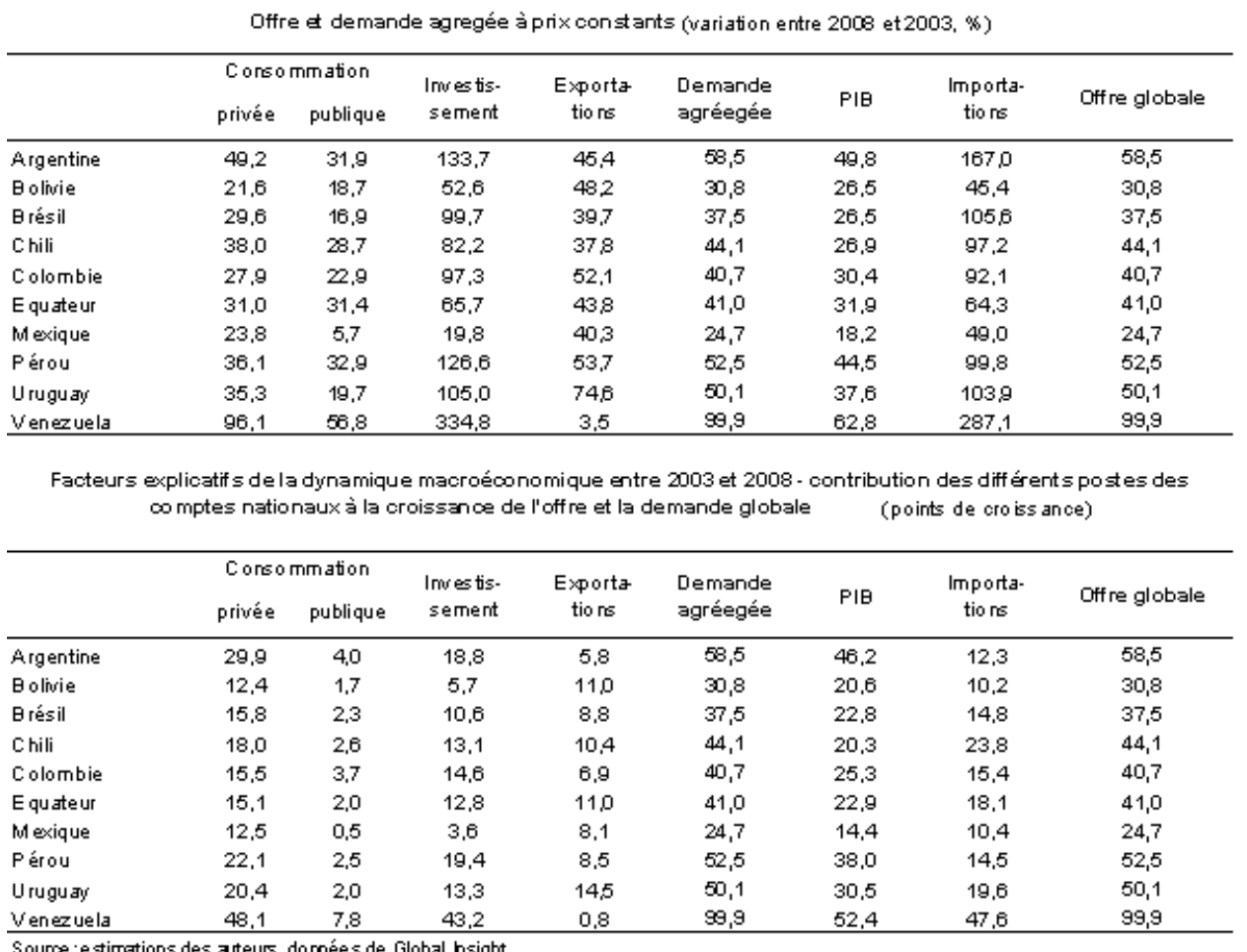

Source : estimations des auteurs, do mées de Global hsight 
Gráfico 3 : Descomposición del crecimiento del PIB (2003-2008, \% de crecimiento)

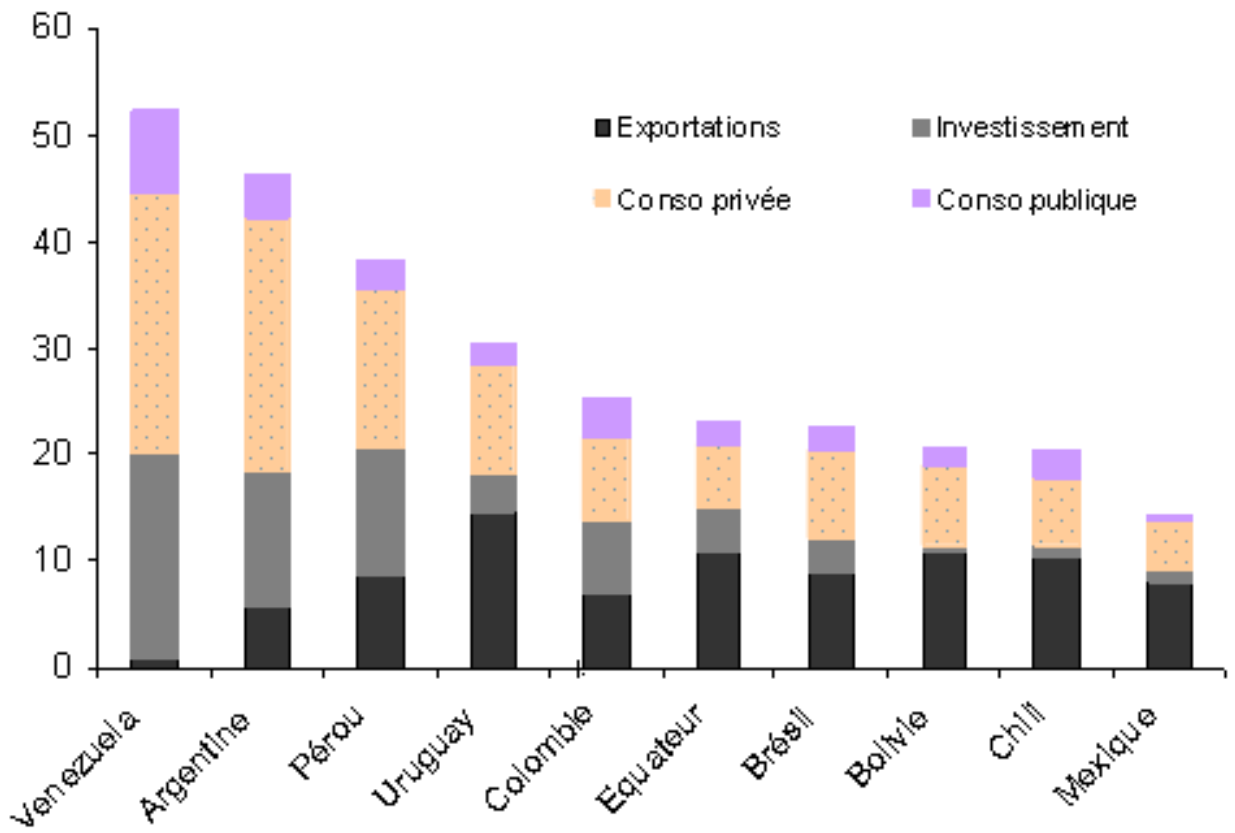

NB : nous awons ret iré de la consomma ion privé et de l'invest issernent la composarte import ée. Faute de st atistiques detaillées, nous avons fait l' hyp othèse que les import a ions comestpondent à 50 \% à chacune de ces deux composantes de la demande domestique.

6 Otra característica destacada de la fase de crecimiento de la primera década de este siglo se refiere a que dicha fase ha estado acompañada de la disminución de la vulnerabilidad financiera de la mayoría de países de la región. Así, en los años 2000, muchos de ellos han experimentado una mejora en los saldos de las finanzas públicas y una disminución de los ratios de endeudamiento público y externo acompañadas de un refuerzo de la posición financiera exterior gracias a un aumento considerable de las reservas internacionales (Ver Gráficos 4,5 y 6).

Gráfico 4 : Saldos presupuestarios

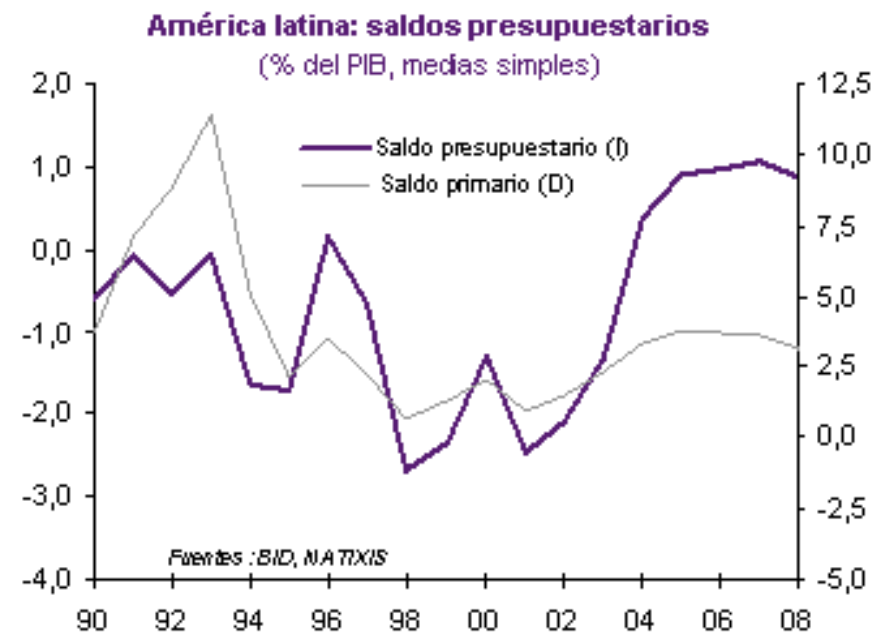




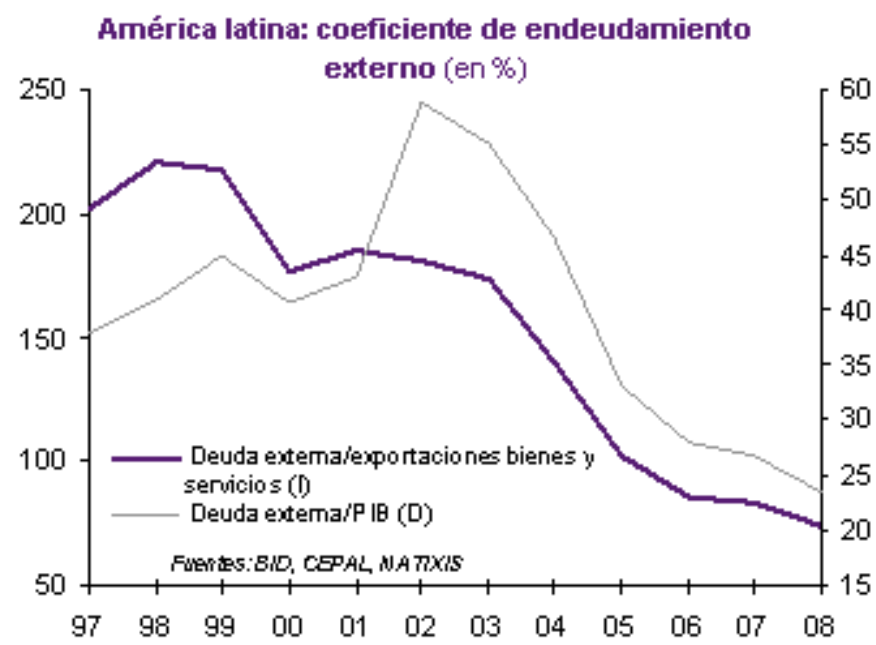

Gráfico 6 : Reservas de divisas

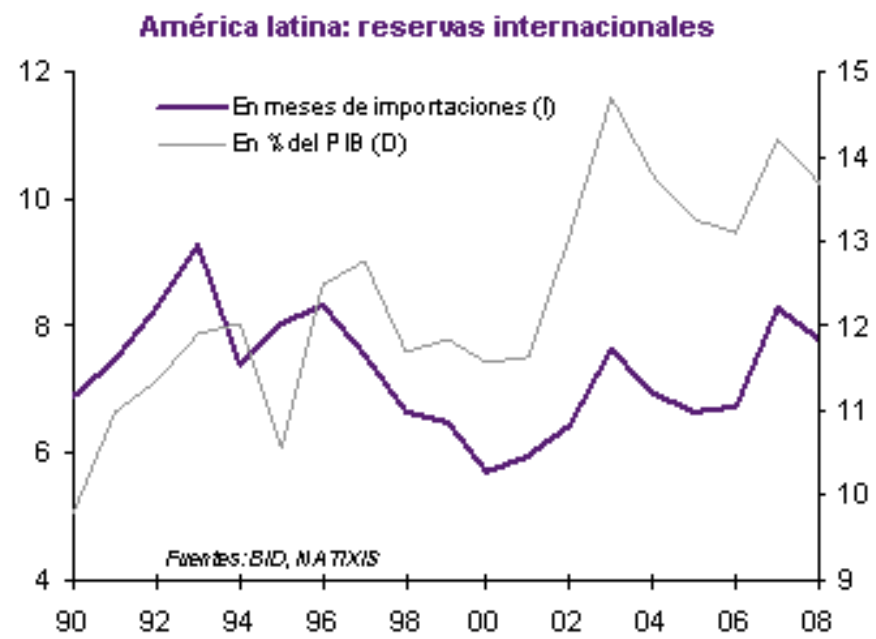

\section{Los efectos negativos de la crisis internacional}

La crisis económica global interrumpió el ciclo de crecimiento 2003-2008. No en vano, la crisis internacional empezó a golpear con fuerza a América Latina a partir de septiembre de 2008 provocando una reacción casi instantánea de las variables financieras, aunque el efecto fue, a fin de cuentas, de corto alcance (Baduel, Ordoñez y Quenan, 2010) : en su mayoría, dichas variables se recuperaron rápidamente a partir del segundo trimestre de 2009 y casi ningún país tuvo que hacer frente a una restricción prolongada de la financiación exterior. Así, entre septiembre de 2008 y marzo de 2009, se registró un aumento generalizado de las primas de riesgo de las deudas soberanas seguido, desde marzo de 2009, de una recuperación de la situación gracias a la disminución de la aversión al riesgo y a una liquidez global abundante. Ello indujo la vuelta de los flujos de capital a la región y un acceso más fácil a los mercados 
internacionales de crédito para la mayoría de países latinoamericanos. Por otra parte, las monedas de los países de la región acusaron una sensible depreciación entre finales de 2008 y principios de 2009 seguida, desde marzo-abril de 2009, de una reevaluación generalizada. Paralelamente, tras la profunda depresión de las bolsas locales a finales de 2008, se constató, a partir de marzo de 2009, una rápida reactivación del dinamismo de la mayoría de centros financieros latinoamericanos.

Los efectos negativos de la crisis internacional se hicieron sentir igualmente, y sobre todo, en el ámbito comercial. Así, en América Latina la crisis se tradujo en una fuerte degradación de los intercambios comerciales tanto en términos de volumen como de valor. En volumen, la CEPAL evalúa la disminución de las exportaciones regionales en un $9 \%$ en 2009. Fruto principalmente del hundimiento de la demanda internacional (sobre todo de los países industrializados), se registró una fuerte caída de las cantidades exportadas, especialmente en los países exportadores de bienes manufacturados que tenían a Estados Unidos como cliente principal (México, Centroamérica). En cuanto a los países exportadores de productos básicos, la caída del volumen exportado fue menor gracias, sobre todo, al mantenimiento de la demanda china. Con respecto al comercio en valor, entre finales de 2008 y principios de 2009, los importes facturados se vieron mermados por una brutal corrección a la baja de los precios de los productos básicos, afectando directamente a los países exportadores netos de materias primas. En cambio, para los países importadores netos, la bajada de precios permitió reducir las tensiones en el saldo corriente en un contexto de restricción crediticia internacional. Pese a todo, el precio de ciertas materias primas clave para algunas economías latinoamericanas (petróleo, cobre y algunos productos agrícolas) registró una notable subida a partir de principios de 2009. El aumento de estos precios supuso un alivio para los países exportadores de materias primas que tienen un acceso limitado a los mercados internacionales de capitales (Venezuela, Argentina) y cuyas finanzas públicas dependen considerablemente de estos productos (México y Ecuador, por ejemplo). Por tanto, a pesar de que los términos del intercambio empeoraron durante todo el año 2009, el deterioro fue menos severo de lo previsto inicialmente. Por otra parte, la disminución de las exportaciones en valor registrada a lo largo del año no provocó un deterioro considerable de los saldos de las balanzas comerciales de las principales economías de la región dado que las importaciones cayeron debido a la recesión.

9 La crisis también ha afectado a América Latina deteriorando el saldo de otras partidas de la balanza por cuenta corriente. Es el caso de las remesas de los emigrantes, que representan una parte no desdeñable del PIB de algunos países de la región (en particular, de México, los pequeños países de Centroamérica y el Caribe o Ecuador) (Gráficos $7 \mathrm{a}$ y $7 \mathrm{~b}$ ). Pese a que estas transferencias son menos volátiles que otros flujos financieros, los efectos de la crisis en los países desarrollados (sobre todo en Estados Unidos y España) y las consecuencias en sus mercados de trabajo provocaron la caída de estos envíos realizados por los inmigrantes latinoamericanos a sus países de origen. Según estimaciones del Banco Interamericano de Desarrollo, estos flujos cayeron en un $15 \%$ en $2009^{3}$. 
Gráfico 7a : América Latina: transferencias realizadas por trabajadores migrantes

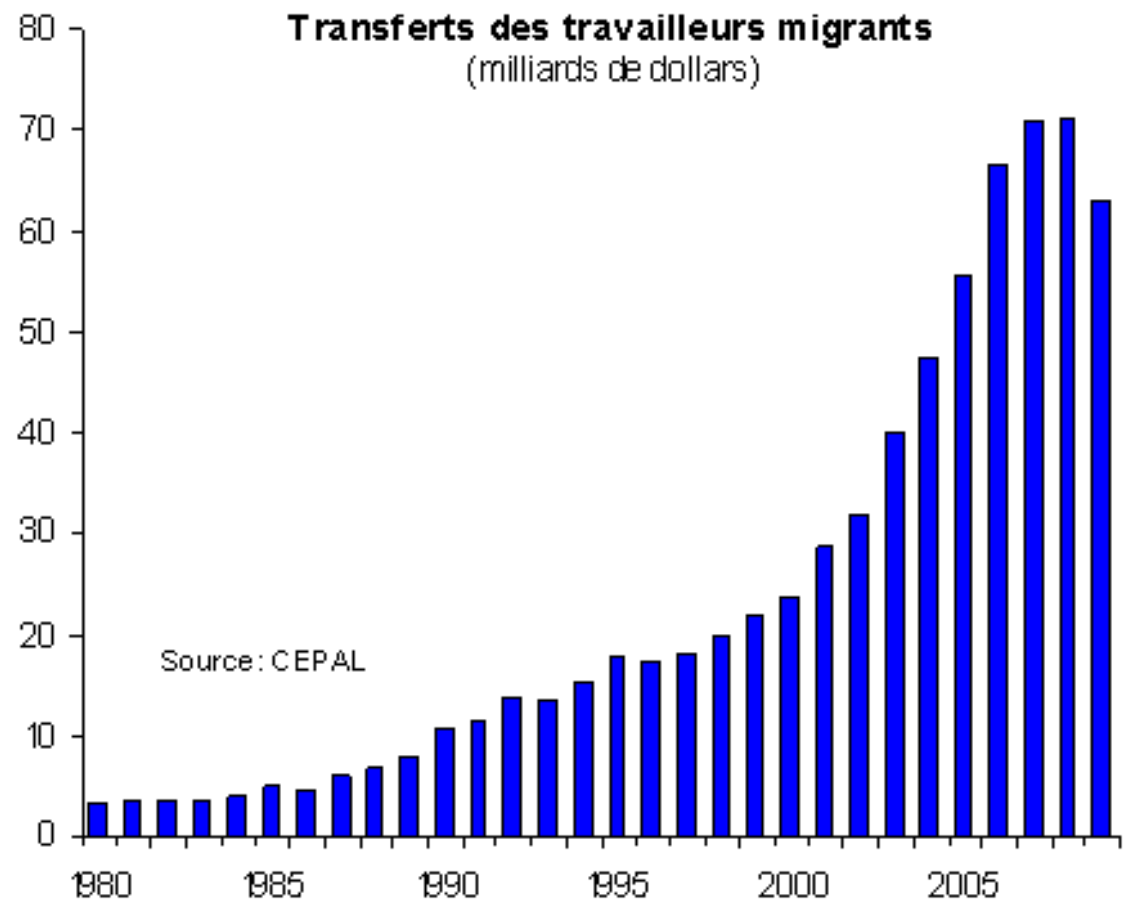

Gráfico $7 \mathrm{~b}$ : Transferencias de trabajadores migrantes

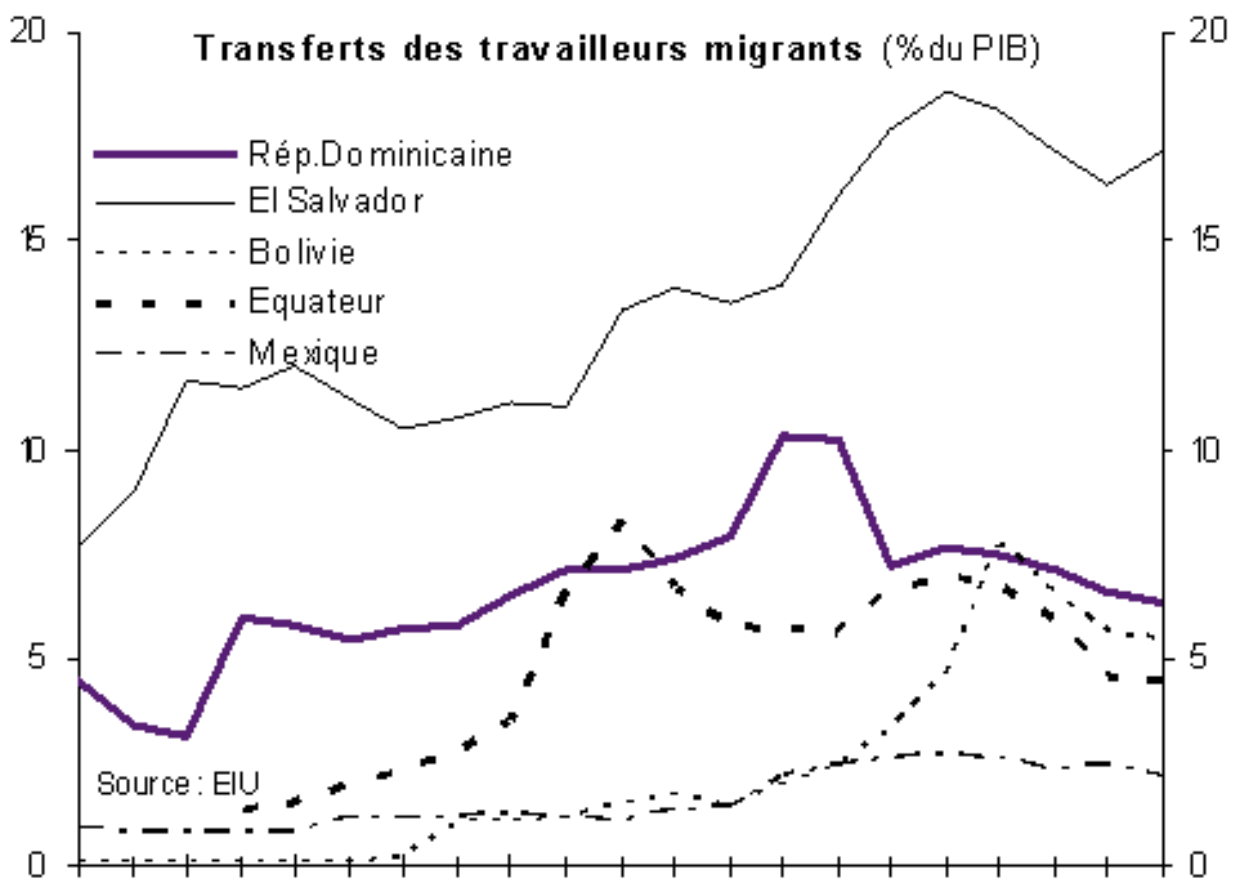

$9091929394959697989900 \quad 0102030405 \quad 0607 \quad 0809 \quad 10$ 


\section{El impacto en el crecimiento regional}

Las perturbaciones resultantes de la crisis internacional provocaron en 2009 una recesión, a fin de cuentas, moderada (disminución del PIB en casi un $2 \%$ ) : la región se ha visto relativamente poco afectada por la crisis en comparación con otras zonas del mundo (Gráfico 8). Sin duda, como señalan algunos observadores y analistas (Machinea, 2010), evaluar los efectos de la crisis financiera tomando como referencia los niveles de crecimiento de 2009 podría contribuir a sobreestimar la capacidad de resistencia de la región. Si se evalúa el impacto de la crisis atendiendo a la diferencia entre la dinámica de crecimiento del período 2005-2007 (ciclo de fuerte crecimiento en la región) y el crecimiento en 2009, América Latina aparece como una de las regiones que más ha sufrido. En 2009, el crecimiento retrocedió en 7 puntos en relación con el crecimiento medio anual del período anterior (Gráfico 9). Sin embargo, esta afirmación debe relativizarse. Por un lado, por un efecto básico:la brusca desaceleración del crecimiento latinoamericano se explica esencialmente por el fuerte dinamismo del crecimiento en los años previos a la crisis. Por otro, evaluar la resistencia de América Latina en su conjunto puede resultar simplista dada la variedad de efectos que la crisis ha tenido en los diferentes países de la región.

11 Mientras algunos países como Brasil registraban un fuerte crecimiento antes de la crisis internacional o han resistido bien frente a la misma, otros se han visto severamente afectados. Es el caso de México, que con un crecimiento que ya era bajo antes de la crisis (inferior a la media regional desde principios de los años 2000), se mostró especialmente vulnerable a las perturbaciones de 2008-2009 debido a la intensidad de sus relaciones económicas con Estados Unidos. México, que representa casi el $30 \%$ del PIB latinoamericano y que en 2009 vivió la mayor recesión de la región (- 6,2\%), ha empañado los resultados económicos de la zona. Si excluimos a México, las consecuencias de la crisis en América Latina son mucho menos dramáticas:el crecimiento regional es nulo en 2009 y su desaceleración, mucho menos significativa (poco más de 5 puntos porcentuales).

Gráfico 8 : Tasa de crecimiento en 2009 ( \% interanual)

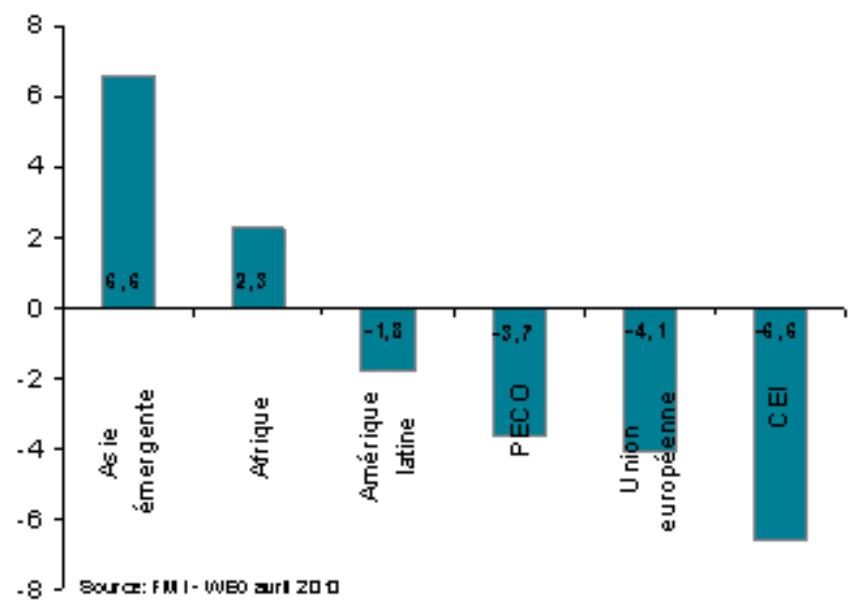




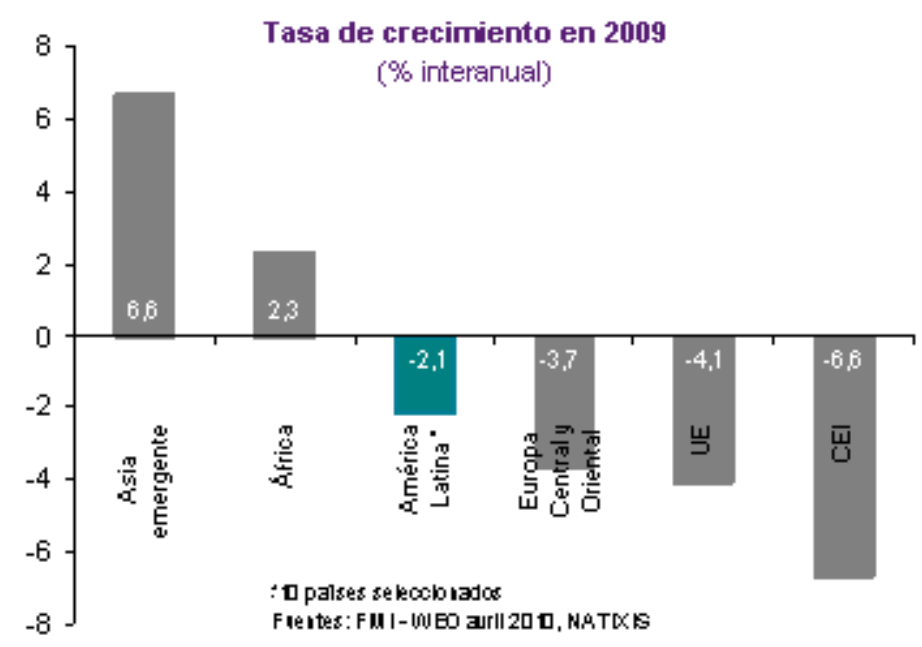

Gráfico 9 : Diferenciales de crecimiento : media 2005-2007 vs. 2009 (en puntos porcentuales)

$-146$

$-9,7$

PECO

$-72$

Amérique latine

$-7[0$

Union européenne

$-60 \quad$ Economies dév eloppées

$-5,4 \quad$ Economies en dév.

$-3,8$

Afrique

$-32$

Asle en dév.

Source: FM I - WEO aril 2010 


\section{Diferenciales de crecimiento: media 2005-2007 vs. 2009 (en puntos porcentuales)}

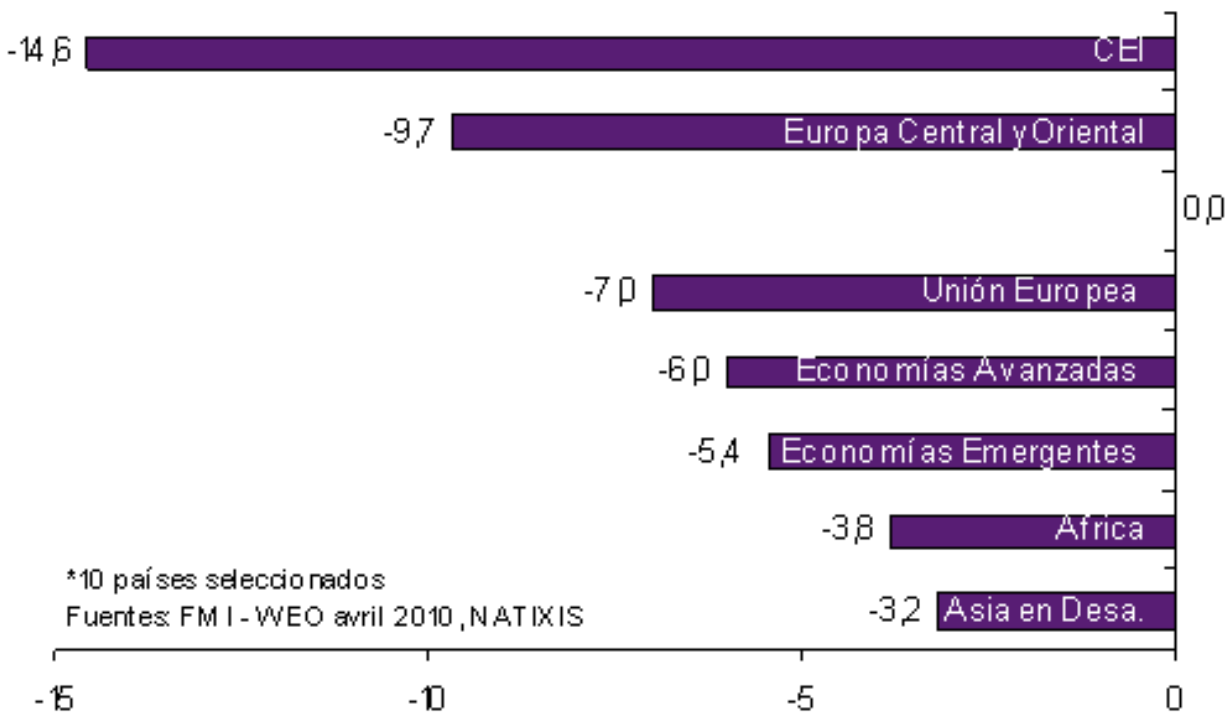

12 Pese a que los shocks externos han tenido un alcance comparable a las que sufrió América Latina durante la crisis de la deuda externa de principios de los 80 (OCDE, 2009), la región ha resistido bien frente a la crisis económica internacional desatada en 2007. Asimismo, ha logrado impedir una recesión aún más profunda y ha podido hacer frente a las turbulencias internacionales sin sufrir - como en el pasado - crisis monetarias o financieras.

Esta capacidad de resistencia a la crisis viene dada por varios factores. Por una parte, el impacto y la importancia de los shocks desfavorables. México, por ejemplo, acusó de lleno los efectos negativos de un shock comercial desfavorable : la contracción de sus exportaciones hacia Estados Unidos - epicentro de la primera fase de la crisis internacional - que constituye, de lejos, su principal mercado, contrasta con una menor disminución de las exportaciones de Brasil, que tiene relaciones comerciales mucho más diversificadas. Además, México se vio muy afectado por la disminución de los ingresos generados por el turismo y las remesas de los emigrantes, como ya hemos señalado.

Por otra parte, la capacidad de respuesta de cada país para contrarrestar las perturbaciones externas depende del grado de libertad del que disponen los poderes públicos. Dado que, como hemos visto, la mayoría de países de la región ha reducido sus fuentes de vulnerabilidad a través de la disminución de los ratios de endeudamiento público y el refuerzo de la posición financiera exterior gracias a un aumento considerable de las reservas de divisas y una mayor solidez de los sistemas bancarios nacionales, los márgenes de maniobra de cada país en el terreno presupuestario han sido decisivos para frenar los efectos recesivos generados por los shocks desfavorables. 


\section{La importancia decisiva de los márgenes de maniobra a nivel presupuestario} disponían los gobiernos de los distintos países de América Latina para aplicar medidas de reactivación anticíclica han sido factores determinantes para atenuar los efectos de los shocks negativos.

En general, los países cuyas finanzas públicas estaban más saneadas han logrado contrarrestar mejor los efectos negativos de la crisis en la economía real (Gráfico 10).

Gráfico 10 : Márgenes presupuestarios y crisis

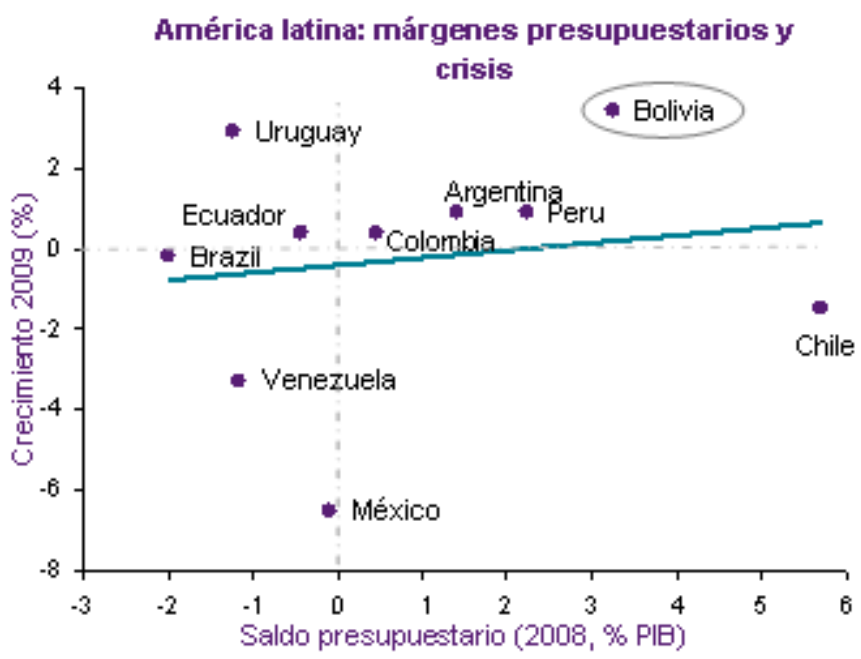

El caso de Bolivia merece especial mención. Este pequeño país (menos del $1 \%$ del PIB de la región) registró en 2009 el crecimiento más dinámico y la desaceleración menos pronunciada ${ }^{4}$. Entre 2003 y 2008, como la mayoría de países de la región, Bolivia se vio favorecida por un ciclo de fuerte crecimiento (alrededor de $5 \%$ de promedio anual ) y un entorno internacional favorable. Sus exportaciones, constituidas sobre todo por hidrocarburos (gas, principalmente) y minerales, se beneficiaron del boom de los precios de los productos básicos. Esta fase de crecimiento estuvo acompañada de una mejora en el superávit de la balanza por cuenta corriente, un importante aumento de las reservas internacionales y una disminución de los indicadores de endeudamiento (externo y público), que permitieron reducir la vulnerabilidad financiera del país y resistir mejor frente a la crisis. En cuanto a la economía real, el elevado nivel de gasto público es lo que explica principalmente el sólido crecimiento de Bolivia. El fuerte aumento del gasto presupuestario (tabla 2) se financió gracias a un importante aumento de los ingresos fiscales, sobre todo tras las reformas del sector de los hidrocarburos de 2005 (que se tradujeron principalmente en aumentos de las tasas de impuestos y royalties percibidos por el Estado) y 2006 (renacionalización del sector de los hidrocarburos, cedido a compañías extranjeras en los años 80-90). Los ingresos presupuestarios, que en 2005 sólo representaban poco más del $30 \%$ del PIB, rozaron el $45 \%$ del PIB entre 2007 y 2009. Así, gracias a las reservas fiscales acumuladas durante el periodo de crecimiento económico y de aumento de precios de los productos exportados, Bolivia fue el único país de la región en registrar un superávit 
presupuestario en 2009 (equivalente al 0,1\% del PIB), y ello a pesar del plan de reactivación lanzado por el gobierno.

Contrariamente a Bolivia, Venezuela es buen ejemplo del fuerte deterioro del estado de las finanzas públicas tras la corrección a la baja de los ingresos del petróleo, que representan alrededor del $50 \%$ de los ingresos fiscales totales. En el marco de un régimen de crecimiento especialmente dependiente del gasto público, la actividad económica acabó acusando notablemente dicha corrección.

Tabla 2 : Evolución de las finanzas públicas

\section{Tabla 2}

América latina finanzas públicas (拖 del PIB)

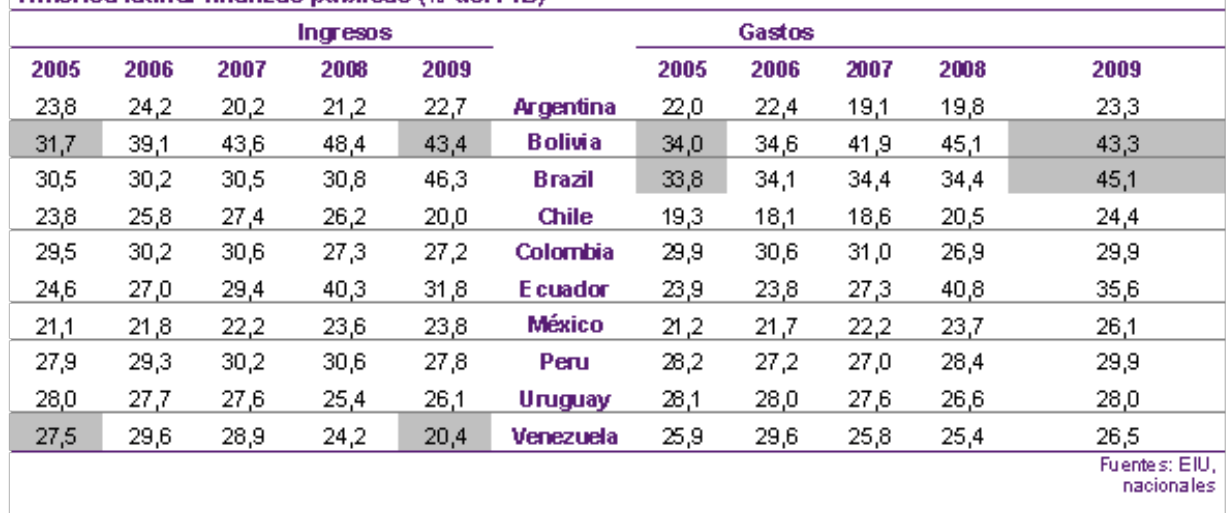

Por otra parte, otros países como Chile o Perú trataron de contrarrestar las tensiones recesivas gracias a los fondos anticíclicos creados previamente. Estos países dedicaron alrededor del $3 \%$ del PIB a políticas presupuestarias expansionistas (Gráfico 11). Pese a todo, Chile, que se caracteriza por tener una economía muy abierta, ha vivido una recesión muy severa.

Gráfico 11 : Estimación de los costos de los planes anticrisis adoptados en 2009 (\% PIB)

América latina: estimación de los costos de los planes anticrisis adoptados en 2009 ( $\%$ PIB)

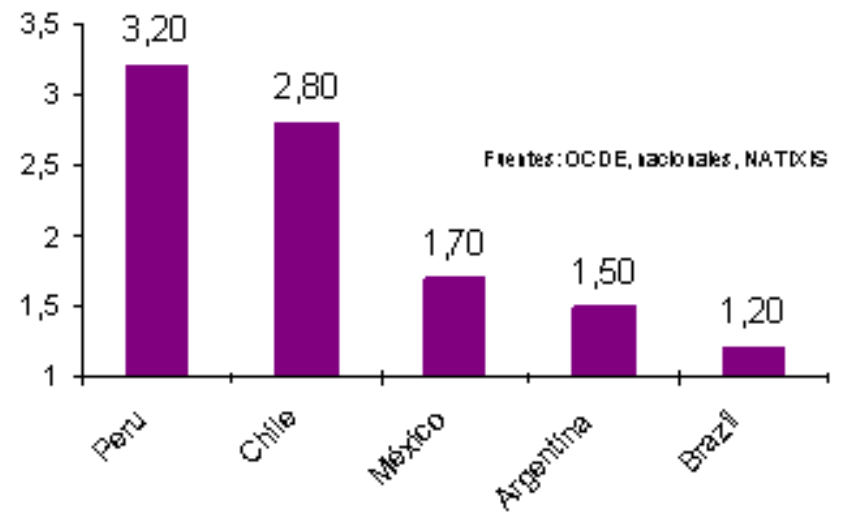

Entre las grandes economías de la región, la capacidad de resistencia a la crisis ha sido particularmente diferente entre Brasil y México (Quenan y Torija-Zane, 2011). Ambos 
países presentan, sin embargo, una similitud importante : sus medidas de estímulo presupuestario han sido de escaso calado comparadas no sólo con las aplicadas por los países desarrollados sino también con las de los países latinoamericanos (ver Gráfico 11).

21 En el caso de Brasil, que no había constituido un fondo anticíclico importante, las medidas de estímulo se financiaron con la disminución moderada del excedente primario. El refuerzo del Plan de Aceleración del Crecimiento (PAC) - lanzado en 2007 con el objetivo de aumentar las inversiones en infraestructuras -, la dependencia limitada de los ingresos fiscales con respecto a los precios de las materias primas y el bajo grado de apertura de una economía cuyo dinamismo reside sobre todo en la demanda interna, se conjugaron para atenuar el impacto recesivo de la crisis internacional.

En el caso de México, como hemos visto, los shocks externos fueron especialmente virulentos, aunque a ello se unieron también unas políticas públicas anticíclicas de alcance limitado. Ciertamente, en los años 2000 mejoraron las cuentas públicas, pero dicha mejora tuvo una base precaria : en efecto, los ingresos y los gastos del Estado siguen siendo fuertemente pro-cíclicos debido a su dependencia de los ingresos del petróleo, lo cual disminuye los márgenes de maniobra de la política fiscal. Es indispensable poner en marcha reformas cuyo objetivo sea reducir la dependencia respecto del petróleo de los ingresos presupuestarios. Sin embargo, estas reformas se enfrentan a una fuerte resistencia .

\section{Conclusión}

La región latinoamericana ha resistido bien en las primeras fases de la crisis económica internacional desatada en 2007 : así, durante el periodo de empeoramiento de la crisis en 2008-2009, los países de América Latina no han vivido crisis bancarias o financieras y han sufrido una recesión más bien moderada.

Este hecho se explica en buena parte por la disminución de la vulnerabilidad financiera durante la fase de expansión del crecimiento entre 2003-2008, así como por la solidez creciente de los sistemas bancarios. Por otra parte, la buena salud de las finanzas públicas ha permitido la rápida puesta en marcha de estímulos presupuestarios. Pese a que dichos estímulos han tenido un alcance menor en comparación con los de los países desarrollados, han contribuido - combinados a menudo con políticas monetarias expansionistas - a atenuar las consecuencias de la recesión.

Ciertamente, el empeoramiento moderado de los saldos presupuestarios ha elevado los indicadores de deuda pública (Gráfico 12) y puede contribuir a reducir los márgenes de maniobra para futuras políticas de reactivación de la demanda interna. Pero las finanzas públicas están bajo control tras varios años de mejora y el incremento de deuda soberana ha sido finalmente pequeño. Por tanto, América Latina no forma parte, a corto plazo, de las zonas y países que pueden vivir graves crisis asociadas a la deriva del endeudamiento público como sí se ha visto a partir de 2010 en varios países europeos. 


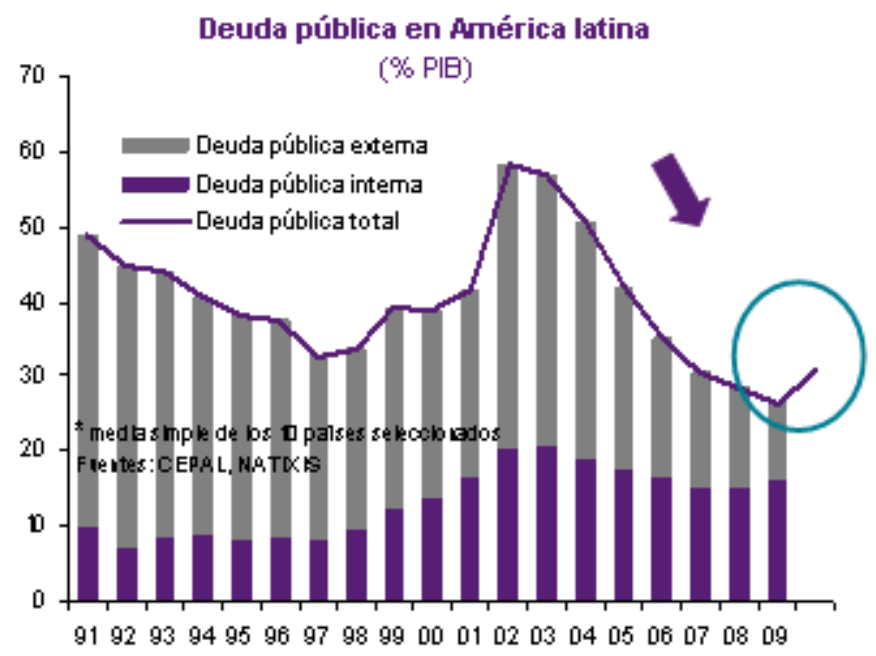

Sin embargo, esta evolución general abarca una gran variedad de situaciones nacionales. Si bien las crisis que se han producido en América Latina debido a los shocksexternos de 2008-2009 pueden considerarse " coyunturales ", no es menos cierto que dichas crisis han puesto de manifiesto la diversidad de trayectorias económicas nacionales que caracteriza a la región.

Así, como lo hemos visto, dos economías extremadamente dependientes de los ingresos deriviados de exportaciones de hidorcarburos, Bolivia y Venezuela, tuvieron comportamientos extremadamente diferentes. Mientras que en Bolivia el incremento de la captación de la renta proveniente de los hidrocarburos va de la mano con una mejora significativa de la situación de las finanzas públicas que ha acrecentado el margen de maniobra para la política contra-cíclica del gobierno, en el caso de Venezuela la economía cayó en la recesión. La crisis internacional ha revelado el carácter pro-cíclico de las finanzas públicas y la fragilización de la economía venezolana, la única entre las grandes economías de la región que no experimentó una reactivación en 2010.

Entre los grandes países de la región, la distinción parece muy clara, como hemos visto, entre Brasil y México. En el caso de Brasil, la diversificación de sus relaciones económicas externas, la baja dependencia de los ingresos fiscales con respecto a los precios de las materias primas y el bajo grado de apertura de la economía han permitido limitar los efectos negativos de las perturbaciones externas. Y lo que es más : dichas perturbaciones se han producido en un marco no sólo de disminución de la vulnerabilidad financiera sino también de mayor crecimiento, el cual, durante el periodo 2003-2008, ha progresado a un ritmo bastante superior al de los años 90 . Si bien el entorno internacional favorable ha sido un factor de crecimiento, éste ha estado respaldado sobre todo por el dinamismo de la demanda interna. Así, antes de que los efectos de la crisis económica se dejaran sentir, Brasil registraba una fase de expansión de la demanda interna que volvió a surgir en 2010, coincidiendo con una nueva aceleración del crecimiento. Más allá de la importancia del aumento del crédito al consumo, todo ello es el reflejo sobre todo de una dinámica de impulso de la demanda interna puesta en marcha desde hace varios años, acompañada del aumento de la renta per cápita, la evolución favorable del mercado de trabajo y el desarrollo de la clase 
media. A todo ello se añaden otros factores como el papel y la influencia crecientes de la diplomacia económica brasileña en espacios como el G20 o los BRICS donde defiende, junto a otros países, la necesidad de reformar la arquitectura financiera internacional. Todo ello permite plantear que Brasil ha vivido una « crisis buena » en 2008-2009.

Sin embargo, los retos a los que debe hacer frente el régimen de crecimiento brasileño son considerables (Miotti, Quenan y Torija-Zane, 2012). Tras la fuerte depreciación sufrida por el real brasileño entre fines de 2008 y comienzo de 2009 la tendencia a la apreciación de la moneda nacional se ha afirmado. Esto, junto con el aumento de los costos salariales y las insuficiencias de la infraestructura física, - en especial a nivel de puertos y aeropuertos - supone el gran desafío de implementar una considerable reestructuración de la oferta. Tras el rebote producido en 2010, estos factores, aunados a la debilidad relativa de la inversión y del ahorro nacional, explican el debilitamiento del crecimiento a partir de 2011. A su vez, la pérdida de dinamismo de la industria nacional - amenazada por la creciente competencia china-, revela el problema de competitividad que aqueja la economía brasileña.

En el caso de México, la crisis internacional ha puesto de manifiesto algunas de las debilidades más evidentes de su régimen de crecimiento. Como hemos visto, el país se ha mostrado especialmente vulnerable a los shocks desvaforables resultantes sobre todo de sus intensas relaciones económicas con Estados Unidos, epicentro de la fase inicial de la crisis. Pese a todo, es preciso señalar que la solidez del sistema bancario y la mejora de la gestión macroeconómica que se ha producido en México desde los años 90 han contribuido a reducir las fuentes de vulnerabilidad y han impedido que dichos shocks hayan originado una crisis financiera.

31 Ahora bien, en el caso de México, al reducido grado de diversificación de sus relaciones económicas internacionales se añade el carácter limitado de las políticas públicas de estímulo presupuestario, en un país donde los ingresos y los gastos del Estado son extremadamente pro-cíclicos debido a su dependencia de las rentas del petróleo. De hecho, dicha demanda tiene dificultades para convertirse en factor de compensación del debilitamiento de la demanda externa. Dado el desarrollo de las maquiladoras (industrias de ensamblaje que producen para el mercado estadounidense) y la aplicación del Tratado de Libre Comercio de América del Norte (TLCAN, firmado por Canadá, Estados Unidos y México), el régimen de crecimiento mexicano está basado en buena medida en exportaciones favorecidas por una mano de obra de bajo costo (en comparación con los salarios de Estados Unidos). Ello genera pocos incentivos para la creación de empleos de calidad y bien remunerados y debilita las fuentes de crecimiento nacionales. Por consiguiente, los desafíos para la economía mexicana son múltiples. Reequilibrar el régimen de crecimiento para darle más peso a la dinámica interna pasa, entre otras, por acrecentar la capacidad de acción del Estado. Por ejemplo, la baja carga tributaria justifica una reforma fiscal que contribuya a darle mayores márgenes de maniobra a las políticas públicas.

La primera fase de la crisis económica internacional ha servido, por tanto, para poner de relieve no sólo los principales rasgos comunes de la región sino también la diversidad de trayectorias económicas de los países latinoamericanos. 


\section{BIBLIOGRAPHIE}

Baduel, Bénédicte, Daniela Ordóñez y Carlos Quenan, Note Mensuelle Amérique latine, Natixis, Paris, janvier 2010.

Machinea, José Luis, El impacto de la crisis en América Latina : una mirada de corto y largo plazo, Universidad de Alcalá - Instituto de Estudios Latinoamericanos, Madrid, 2010.

Miotti, Luis, Carlos Quenan y Edgardo Torija-Zane, « Continuités et ruptures dans l'accumulation et la régulation en Amérique latine dans les années 2000 : les cas de l'Argentine, du Brésil et du Chili »,in Revue de la Régulation 11, Paris, 1er semestre 2012.

Ocampo, José Antonio, "The impact of the global financial crisis on Latin America ", in Cepal Review, $\mathrm{n}^{\circ}$ 98, Santiago du Chili, août 2009.

OECD, Latin America Economic Outlook, Paris, 2009.

Quenan, Carlos y Edgardo Torija-Zane, « Dinámicas económicas : tendencias y perspectivas », in Carlos Quenan y Sebastien Velut, Los desafíos del desarrollo en América Latina. Dinámicas socioeconómicas y políticas públicas, Agence Française de Développement/Institut des Amériques, Paris, 2011.

\section{NOTES}

1. Es el caso de Argentina y Brasil en los sectores agrícola y agroalimentario, de Chile, en el sector del cobre, de Perú con los metales, y de Venezuela y Ecuador en lo que se refiere al petróleo.

2. Esta cifra está subestimada si se incluye la inversión de sectores cuya producción está orientada a la exportación.

3. Según datos de la BID : http://www.iadb.org/news-releases/2010-03/english/remittances-tolatin-america-stabilizing-after-15-drop-last-year-fomin-6671.html.

4. Con un retroceso global de América Latina que, como vimos, fue de casi $-2 \%$, Bolivia registróen 2009 la tasa de crecimiento más elevada de la región, 3,4\%, en las antípodas de la situación de México que como ya se indicó sufrió una caida del PIB de 6,2 \%.

\section{RÉSUMÉS}

La région latino-américaine a bien résisté au cours de la première phase de la crise économique internationale déclenchée en 2007. Pendant la période d'aggravation de la crise de 2008-2009, consécutive à la faillite de Lehman Brothers, les pays d'Amérique latine n'ont pas connu de crises monétaires ou financières et ont subi une récession plutôt modérée et de courte portée car la reprise a été vigoureuse en 2010. Cette capacité de résistance s'explique dans une large mesure par la diminution de la vulnérabilité financière pendant la phase d'expansion de la croissance 2003-2008.

Mais au-delà des caractéristiques communes à l'ensemble de la région, la crise a mis en évidence 
une grande diversité de situations nationales. Les facteurs qui expliquent ces différences ont trait à l'ampleur des chocs défavorables subis par chaque économie, ainsi que le degré de diversification des relations économiques extérieures et les marges de manœuvre de chaque pays pour mettre en place des mesures de relance contre-cycliques. Ceci permet de distinguer les cas du Mexique et du Venezuela, fortement affectés en 2009, par rapport à ceux de la Bolivie ou du Brésil, qui ont bien résisté pendant la première phase de la crise économique internationale. Cependant, même les pays dont les régimes de croissance ont pu atténuer les effets défavorables des chocs négatifs encaissés sont confrontés à des défis importants.

La región latinoamericana ha resistido bien en la primera fase de la crisis económica internacional desatada en 2007. Durante el período de agravación de la crisis de 2008-2009, consecutivo a la quiebra de Lehman Brothers, los países de América Latina no han vivido crisis monetarias o financieras y han sufrido una recesión más bien moderada y de corta duración ya que la recuperación fue vigorosa en 2010. Esta capacidad de resistencia se explica en buena medida por la disminución de la vulnerabilidad financiera durante la fase de expansión del crecimiento 2003-2008.

Pero más allá de las características comunes al conjunto de la región, la crisis ha puesto de manifiesto una gran diversidad de situaciones nacionales. Los factores que explican estas diferencias tienen que ver con la amplitud de los shocks desfavorables sufridos por cada economía, así como el grado de diversificación de las relaciones económicas externas y los márgenes de maniobra de cada país para aplicar medidas de reactivación anti-cíclica. Esto permite distinguir los casos de México y Venezuela, duramente afectados en 2009, de los de Bolivia o Brasil, que resistieron bastante bien en la primera fase de la crisis económica internacional. Sin embargo, aun los países cuyos regímenes de crecimiento han podido atenuar los efectos desfavorables de los shocks negativos en esta fase están confrontados a retos importantes.

The Latin American region held up well during the first phase of the international economic crisis started in 2007. During the period of worsening of the crisis, after the collapse of Lehman Brothers, Latin American countries did not know any monetary or financial crisis and experimented a mild recession because the recovery was strong in 2010. This degree of resilience is explained to a large extent by the decrease of financial vulnerability during the phase of growth expansion (2003-2008).

However, beyond the common features to the region as a whole, the crisis has exposed a wide range of national situations. The factors that lie behind these differences are related to the magnitude of the unfavourable shocks affecting each economy, the degree of diversification of the external economic relations and the manoeuvring room of each country to implement counter-cyclical measures. This helps to distinguish the cases of Mexico and Venezuela, heavily affected in 2009, compared with those of Bolivia or Brazil, which held up well during the first phase of the international economic crisis. Nevertheless, even the countries whose growth regimes may have mitigated the unfavourable effects of the adverse shocks suffered serious challenges. 


\section{INDEX}

Keywords : Latin America, international crisis, external shocks, financial vulnerability, growth, recession, counter-cyclical policies

Palabras claves : América Latina, crisis internacional, shocks externos, vulnerabilidad financiera, crecimiento, políticas anti-cíclicas

Mots-clés : Amérique latine, crise internationale, chocs externes, vulnérabilité financière, croissance, politiques contre-cycliques

\section{AUTEUR}

\section{CARLOS QUENAN}

Carlos Quenan est professeur des universités en sciences économiques à l'Institut des Hautes Études de l'Amérique latine (IHEAL, Université de Paris III Sorbonne Nouvelle). Il est également chercheur au CREDA - Unité mixte de recherche Université Sorbonne Nouvelle/CNRS -, où il est responsable de l'axe « Intégration régionale».

Vice-président Amérique latine à l'Institut des Amériques, il est également co-président de la Section Europe/Amérique latine de la Latin American Studies Association (LASA).

Il est auteur ou co-auteur d'une soixantaine de livres et de nombreux articles scientifiques et de divulgation, notamment dans le domaine des relations économiques internationales, des relations Europe/Amérique latine, de l'intégration régionale et de la macroéconomie et des politiques économiques des pays émergents, principalement latino-américains.

En outre, il a été ou est professeur invité dans de nombreuses institutions universitaires et de recherche d'Amérique latine, États-Unis, Canada, Europe et Afrique, et est membre du comité éditorial de nombreuses revues et publications spécialisées et de diverses instances internationales de recherche.

Il a été ou est consultant auprès de nombreuses institutions publiques internationales, nationales des pays en développement, ainsi qu'auprès d'instances publiques et privées en Europe et en France (notamment consultant permanent au service de la recherche économique à Natixis). quenanatwanadoopointfr 